\title{
Features for making decisions in the interests of organizing transport processes
}

\author{
G. I. Moskvitin, A. B. Pismennaya, P. N. Abroskin, and V. V. Korsakova
}

Federal State Autonomous Educational Institution for Higher Education "Russian University of Transport", Moscow, Russia

\begin{abstract}
The practice of management activities suggests that the experience and knowledge of managers alone cannot always provide an optimal or even rational solution without additional scientifically grounded methods for assessing the effectiveness of possible options for organizing transport processes. All this generated a need for the development of scientific methods for making (developing, supporting, justifying) decisions that would make it possible to develop specific recommendations for the head who manages the facility in difficult situations. These methods included the formation of effectiveness measures in the form of maximum or minimum of a single indicator - the target function with or without constraints and various algorithms for finding the values of arguments that provide the required value of this function. Subsequently, mathematical methods for solving multi-criteria problems involving different goals of the operation and using, respectively, a lot of alternative effectiveness measures began to develop.
\end{abstract}

\section{Introduction}

The article presents approaches to solving problems with fuzzy decision conditions. In this case the exact values of $v \in \mathrm{N}$ choice conditions are not set, as in the problem solution in the conditions of definiteness, and the distribution laws $\mathrm{P}(v)$ of random variables $v$ on the set $\mathrm{N}$ are not known, as in the problem solution in the conditions of risk. However, unlike problems with fuzzy conditions, the range of possible values of the input data is given as a set that has clearly defined boundaries in the set of admissible values of these quantities. Put it in other way, the input data under conditions of transport process uncertainty can be represented as a clear set of possible values of the corresponding physical quantities, which is a subset of the set of admissible values of these quantities. In problems with fuzzy conditions, a set of input data is specified as a fuzzy set of possible values, which is also a subset of their admissible values.

Despite the adequacy of fuzzy initial conditions to the real problems of transport management decisions and some advantages of computational procedures, it should be noted that at present the possibility of practical application of problems with fuzzy conditions is very limited.

This is due to the following reasons: 
- Lack of common effective methods and procedures for determining the membership functions for each fuzzy set of multiple data, which characterize the management objective and the initial situation for developing a transport management decision;

- High time consumption in determining such membership functions, which reduces the efficiency of decision-making process;

- Influence of subjective, often erroneous expert opinion in determining the values of the membership functions, which may ultimately lead to an overall erroneous decision;

- Insufficient development of the majority of methods for solving problems with fuzzy conditions, primarily in the direction of their practical application to solve complex specific problems of transport-management decisions in real-world conditions.

For wider implementation of achievements of the prospective theory of fuzzy sets in the practice of decision-making, it is necessary to: 1) make a detailed analysis of transport process control tasks with possible fuzzy initial data, formulate them in terms of fuzzy sets and select appropriate methods for solving the task set with fuzzy initial conditions; 2) make practical testing and refinement of selected methods for solving tasks with fuzzy conditions to ensure decision-making processes in transport process conditions; 3 ) develop automated methods that provide operational and effective based on the selected methods.

\section{Materials and methods}

Problems with fuzzy conditions, methods for solving which are described in the domestic and foreign literature, can be divided into two groups:

- one-step static mathematical programming problems;

- multistep dynamic decision-making problems (fuzzy dynamic programming problems).

One-step static problems of fuzzy mathematical programming can be formulated on the basis of a general optimization problem with fuzzy conditions. Such a problem is defined as follows: find a solution $\mu^{*} \in \mathrm{M}$, wherein

$$
\begin{aligned}
& G *=g(\mu *)=\operatorname{maxg}(\mu) \\
& \varphi i(\mu) \leq 0, i=1,2, \ldots, n
\end{aligned}
$$

The conditions for choosing $v \in \mathrm{N}$ are omitted here, but it is assumed that they are taken into account in the functions $\mathrm{g}(\mu)$ and $\varphi_{\mathrm{i}}(\mu)$. In this case we have well-defined functions $\mathrm{g}$ and $\varphi_{\mathrm{i}}$, a certain goal (to calculate the exact value $\mu$, maximizing the function $\mathrm{g}(\mu)$ ) explicit constraints, and an explicit set $\mathrm{M}$ of admissible values of the choice parameters $\mu$. However, under real conditions, only fuzzy descriptions of the functions $g$ and $\varphi_{i}$, the variables on which they depend, and the set M itself may be at our disposal. In this case we will have fuzzy mathematical programming problems.

Depending on combination of the listed clear and fuzzy conditions, different formulations of problems of fuzzy mathematical programming can take place. Let's list some of these formulations and individual approaches to solving problems.

Find a solution $\mu^{*}$, wherein

$$
\begin{gathered}
g *=g(\mu *)=\max g(\mu), \\
\mu \in M
\end{gathered}
$$

where $\mathrm{M}-\mathrm{a}$ fuzzy set with the membership function $\mu_{\mathrm{M}}: \mathrm{M} \rightarrow[0,1]$. 
Put it in other way, in this problem the fuzzy formulated constraints (1) are defined as a fuzzy set $\mathrm{M}$ defining admissible alternatives $\mu$.

To solve such a problem, we propose to consider the normalized function $g_{H}(\mu)=g(\mu) /$ $\sup g(\mu)$ as a membership function of the fuzzy goal. The value of $g_{\mathrm{H}}(\mu)$ of this function is regarded as the degree of goal achievement when choosing the alternative $\mu \in \mathrm{M}$. As a result, the search for a solution is reduced according to the Bellman-Sadeh approach $[1,11,12]$ to the merging of fuzzy sets given by the membership functions $g_{H}(\mu)$ and $\mu_{M}(\mu)$. From the obtained fuzzy solution $R$, select the alternative that has the maximum degree of membership in this solution. As a result, we obtain that the solution to the problem is the value $\mu^{*}$ that provides

$$
\mu R(\mu *)=\max _{\mu \in M} \mu R(\mu)=\max _{\mu \in M} \min \{\mu M(\mu), g H(\mu)\}
$$

Find a value $\mu^{*}$, wherein

$$
g(\mu *) \geq \approx g 0, \varphi(\mu *) \leq \approx 0,
$$

where the wavy lines indicate the fuzziness of the corresponding inequalities.

This problem corresponds to a fuzzy maximum function $g(\mu)$, when one does not want to reach a certain maximum value, but to ensure that $g\left(\mu^{*}\right)$ exceeds some fuzzy value $\mathrm{g} 0$. In addition, the constraints are also given as fuzzy inequalities, i.e., the value $\varphi\left(\mu^{*}\right)$ can be approximately less than zero.

One possible approach to a more detailed formalization of such fuzzy tasks is as follows. Let $g_{0}$ be a given value of function $g(\mu)$,achievement of which is considered sufficient to solve the problem, and let there be (for example, given by LPR) two threshold levels a and $\mathrm{b}$, such that inequalities $\mathrm{g}(\mu) \leq \mathrm{g}_{0}-\mathrm{a}$ and $\varphi(\mu) \geq \mathrm{b}$ mean strong violation of inequalities $\mathrm{g}\left(\mu^{*}\right)$ $\geq \mathrm{g}_{0}$ and $\varphi\left(\mu^{*}\right) \leq 0$. Then the fuzzy goals and constraints can be defined with the corresponding fuzzy sets A and B having the following membership functions:

$$
\begin{aligned}
& \mu A(\mu)=\left\{\begin{array}{c}
0, \text { if } g(\mu) \leq g 0-a ; \\
\alpha(\mu, a), \text { if } g 0-a<g(\mu)<g 0 ; \\
1, \text { if } g(\mu) \geq g 0 ;
\end{array}\right. \\
& \mu B(\mu)=\left\{\begin{array}{c}
0, \text { if } \varphi(\mu) \geq b ; \\
\beta(\mu, a), \text { if } 0<\varphi(\mu)<b ; \\
1, \text { if } \varphi(\mu) \leq 0 ;
\end{array}\right.
\end{aligned}
$$

where $\alpha$ and $\beta-$ some membership functions $\mu \rightarrow[0,1]$, describing the degree to which the inequalities are fulfilled from the point of view of the decision maker.

As a result, the problem is formulated using fuzzy goals and constraints, for the fusion of which the Bellman - Sadeh approach is applied.

Find an optimal solution $\mu^{*}$ for a fuzzy function $\mathrm{g}(\mu)$ characterized by an identity function $\mu_{\mathrm{g}}: \mathrm{M} \times \mathrm{G} \rightarrow[0,1]$, and fuzzy constraints defined by an identity function $\mu_{\mathrm{M}}: \mathrm{M} \rightarrow[0,1]$, where $G$ - the set of possible values of function $g(\mu)$. In the special case $G=R^{1}$, where $R^{1}$ - the set of real numbers [8].

Find a solution $\square *$, wherein

$$
g *=g(\mu *)=\max g(\mu) ; \varphi i(\mu) \leq 0, i=1,2, \ldots, n,
$$

where $\varphi_{\mathrm{i}}(\mu)$ - a function with fuzzy parameters. 
For example, in case of linear functions,

$$
\varphi \operatorname{\varphi i}(x)=\sum a i j+b i, i=1,2, \ldots, n,
$$

each parameter $a_{i j}, b_{i}$ is described by corresponding fuzzy sets with membership functions $\mu_{\mathrm{aij}}$ and $\mu_{\mathrm{bi}}$.

The multistep dynamic decision-making problems are analogous to dynamic optimal control problems under clear conditions. To solve such problems, R. Bellman and L. Zadeh developed approaches based on the application of dynamic programming methods $[1,12]$.

The problem with explicit conditions, for the solution of which the method of dynamic programming is used, is formulated as follows.

The functioning of the control object at each time interval $\left(t_{i-1}, t_{i}\right], i=1,2, \ldots, n$, is described by the transition function

$$
z_{\mathrm{i}}=\psi\left(z_{\mathrm{i}}-1, u_{\mathrm{i}}\right), z_{\mathrm{i}} \in Z_{\mathrm{i}} \subset Z, u_{\mathrm{i}} \in U_{\mathrm{i}} \subset U,
$$

where $\mathrm{z}_{\mathrm{i}}, \mathrm{z}_{\mathrm{i}-1}$ - states of the object at moments $\mathrm{t}_{\mathrm{i}}$ and $\mathrm{t}_{\mathrm{i}-1}$;

$\mathrm{u}_{\mathrm{i}}$ - control action on the object on the interval $\left(\mathrm{t}_{\mathrm{i}-1}, \mathrm{t}_{\mathrm{i}}\right]$;

$\mathrm{Z}_{\mathrm{i}}$ - the set of admissible states of the object at the moment $\mathrm{t}_{\mathrm{i}}$;

$\mathrm{Z}$ - the set of admissible states on the whole interval $\left(\mathrm{t}_{0}, \mathrm{t}_{\mathrm{n}}\right]$;

$\mathrm{U}_{\mathrm{i}}$ - the set of admissible input actions on the interval $\left(\mathrm{t}_{\mathrm{i}-1}, \mathrm{t}_{\mathrm{i}}\right]$;

$\mathrm{U}$ - the set of admissible input actions over the whole

control interval $\left(t_{0}, t_{n}\right]$.

The index of control efficiency at each interval $\left(t_{i-1}, t_{i}\right]$ is determined by the dependence

$$
g i=g(z i)=g\left[\psi\left(z_{\mathrm{i}}-1, u_{\mathrm{i}}\right)\right]
$$

It is required to determine the optimal sequence of controls $\mathrm{u}_{1}, \mathrm{u}_{2}, \ldots, \mathrm{u}_{\mathrm{n}}$ and the corresponding object states $\mathrm{z}_{1}, \mathrm{z}_{2}, \ldots, \mathrm{z}_{\mathrm{n}}$, ensuring the maximum value of the indicator $\mathrm{g}\left(\mathrm{z}_{\mathrm{n}}\right)$.

Under fuzzy-defined conditions, the decision-maker can have fuzzy functions $\psi$ and $g$, constraints $Z_{i}$ and $U_{i}$ defined as fuzzy sets, a fuzzy-defined maximum function $g\left(z_{n}\right)$, and a fuzzy-defined end time of the process $t_{n}$. Approaches to solving such problems under different conditions are described in [1]. Let's consider one of these problems.

Assigned: transient function of the control object

$$
z_{\mathrm{i}}=\psi\left(z_{\mathrm{i}}-1, u_{\mathrm{i}}\right), z_{\mathrm{i}} \in Z_{\mathrm{i}} \subset Z, i=1,2, \ldots, n,
$$

where $Z_{i}$ - a fuzzy set;

constraints on permissible values of control actions $u_{i} \in U_{i} \subset U$, where $U_{i}$ - fuzzy sets with membership functions $\mu_{\mathrm{i}}\left(\mathrm{u}_{\mathrm{i}}\right)$;

control objective in the form of a fuzzy set $A \subset Z$ with an identity function $\mu_{A}\left(z_{n}\right)$, which is a fuzzy constraint on the state of the object $z_{n}$ at the last moment of time $t_{n}$;

the initial state of the control object $z_{0}$ and the end time of the control process $t_{n}$.

Determine the sequence of control actions $\mathrm{u}_{1}{ }^{*}, \mathrm{u}_{2}{ }^{*}, \ldots, \mathrm{u}_{\mathrm{n}}{ }^{*}$, ensuring the maximum degree of fuzzy goal achievement under fuzzy constraints.

Thus, in this problem, the goal is a fuzzy set $\mathrm{A}$ in contrast to classical dynamic programming problems [3], where the goal is clearly defined as value $z_{n}$ that maximizes the index $g\left(z_{n}\right)$.

Note that the fuzzy goal A can be considered a fuzzy subset of the set $U^{n}=U \times U \times \cdots \times$ $\mathrm{U}$, because the state $\mathrm{z}_{\mathrm{n}} \square$ of $A$ can be expressed as a dependence $\mathrm{z}_{\mathrm{n}}\left(\mathrm{z}_{0}, \mathrm{u}_{1}, \ldots, \mathrm{u}_{\mathrm{n}}\right)$. Then the identity function of the target on the set Un will be 


$$
\mu_{\mathrm{A}} *\left(u_{1}, u_{1}, \ldots, u_{\mathrm{n}}\right)=\mu_{\mathrm{A}}\left(z_{\mathrm{n}}\right)
$$

In accordance with the general Bellman - Sadeh approach, this problem is solved in two stages. At the first stage, the solution is defined as a fuzzy set $\mathrm{R} \subset \mathrm{U} \times \mathrm{U} \times \cdots \times \mathrm{U}=\mathrm{U}^{\mathrm{n}}$ with membership function $\mu_{\mathrm{R}}\left(\mathrm{u}_{1}, \mathrm{u}_{2}, \ldots, \mathrm{u}_{\mathrm{n}}\right)$. At the second, the selection of specific control actions $\mathrm{u}_{1}, \mathrm{u}_{2}, \ldots, \mathrm{u}_{\mathrm{n}}$, having the maximum degree of belonging to the fuzzy solution $\mathrm{R}$, is performed.

As a result,

$$
\begin{gathered}
\mu_{\mathrm{R}}\left(u_{1}, u_{2}, \ldots, u_{\mathrm{n}}\right)=\min \left\{\mu_{\mathrm{A}} *\left(u_{1}, u_{2}, \ldots, u_{\mathrm{n}}\right), \mu_{1}\left(u_{1}\right), \mu_{2}\left(u_{2}\right), \ldots, \mu_{\mathrm{n}}\left(u_{\mathrm{n}}\right)\right\} \\
\text { or } \\
\mu_{\mathrm{R}}\left(z_{\mathrm{n}}\right)=\min \left\{\mu_{\mathrm{A}}\left(z_{\mathrm{n}}\right), \mu_{1}\left(u_{1}\right), \mu_{2}\left(u_{2}\right), \ldots, \mu_{\mathrm{n}}\left(u_{\mathrm{n}}\right)\right\}
\end{gathered}
$$

Now it is necessary to determine the values $\mathrm{u}_{1}{ }^{*}, \mathrm{u}_{2}{ }^{*}, \ldots, \mathrm{u}_{\mathrm{n}}{ }^{*}$, providing $\max \mu_{\mathrm{R}}\left(\mathrm{z}_{\mathrm{n}}\right)$.

To determine the desired values, we will use the usual dynamic programming procedure described in [2], only instead of $g\left(z_{n}\right)$, we will maximize the membership function $\mu_{R}\left(z_{n}\right)$.

In accordance with the principle of optimality $[2,7,9]$, for each $\mathrm{i}$-th interval $\left(\mathrm{t}_{\mathrm{i}-1}, \mathrm{t}_{\mathrm{i}}\right]$ it is necessary to choose such controls $u_{i}, u_{i+1}, \ldots, u_{n}$, which depend only on the object state $z_{i-1}$ at the beginning of this interval and provide the maximum value of the indicator $\mathrm{g}(\mathrm{zn})$. As a result, we obtain the following relation for a well-defined dynamic programming problem [4]:

$$
\begin{gathered}
\left.\left.f_{\mathrm{n}}-(i-1)\left(z_{\mathrm{i}}-1\right)=\max g\left(z_{\mathrm{n}}\right)=\max _{\mathrm{i}}, \ldots, u_{\mathrm{n}} \max _{\mathrm{i}} \quad u_{\mathrm{i}}+1, \ldots, z_{\mathrm{n}}\right)\right]=\max _{\mathrm{n}}-i\left(z_{\mathrm{i}}\right)= \\
=\max _{\mathrm{n}}-i\left[\psi\left(z_{\mathrm{i}}-1, u_{\mathrm{i}}\right)\right] . \\
u_{\mathrm{i}}
\end{gathered}
$$

Replacing (5) $g\left(z_{n}\right)$ with $\mu_{R}\left(z_{n}\right)$, we obtain a similar relation for the considered fuzzy problem:

$$
\begin{gathered}
\left.f_{\mathrm{n}}-(i-1)\left(z_{\mathrm{i}}-1\right)=\max _{u_{\mathrm{i}}, \ldots, u_{\mathrm{n}}} \mu_{\mathrm{R}}\left(z_{\mathrm{n}}\right)=\max _{u_{\mathrm{i}} \quad u_{\mathrm{i}}+1, \ldots, u_{\mathrm{n}}} \max _{\mathrm{i}}\left(z_{\mathrm{n}}\right)\right]=\max f_{\mathrm{n}}-i\left(z_{\mathrm{i}}\right)= \\
=\max f_{\mathrm{n}}-i\left[\psi\left(z_{\mathrm{i}}-1, u_{\mathrm{i}}\right)\right] .
\end{gathered}
$$

$u_{\mathrm{i}}$

Based on this ratio, an analytical or tabular construction of the function $\mathrm{f}_{\mathrm{n}-\mathrm{(}-1-1)}\left(\mathrm{Z}_{\mathrm{i}-1}\right)$ is performed for each $\mathrm{i}$-th interval, $\mathrm{i}=1,2, \ldots, \mathrm{n}$. To reduce the number of options under consideration, the function is constructed from the last interval $i=n$, i.e. from the function

$$
f_{1}\left(z_{\mathrm{n}}-1\right)=\max _{u_{\mathrm{n}}} f_{0}\left(z_{\mathrm{n}}\right)=\max _{u_{\mathrm{n}}}\left(z_{\mathrm{n}}\right) .
$$

Since the search for the maximum is carried out only on the variable un, the identity function of the fuzzy solution (4.14) can be represented as a dependence

$$
\mu_{\mathrm{R}}\left(z_{\mathrm{n}}\right)=\min \left\{\mu_{\mathrm{A}}\left(z_{\mathrm{n}}\right), \mu_{\mathrm{n}}\left(u_{\mathrm{n}}\right)\right\} .
$$

As a result of 


$$
f_{1}\left(z_{n}-1\right)=\max \min \left\{\mu_{A}\left[\psi\left(z_{n}-1, u_{n}\right)\right], \mu_{n}\left(u_{n}\right)\right\}
$$

By trying permissible values of $z_{n}-1$, un, function graph $\mathrm{fl}\left(\mathrm{z}_{\mathrm{n}}-1\right)$ is constructed and the dependence $u_{n}=\varphi_{n}\left(z_{n-1}\right)$ is determined, which provides in accordance with (19) and (20)

$$
\max \mu_{\mathrm{R}}\left[\psi\left(z_{\mathrm{n}}-1, u_{\mathrm{n}}\right)\right] .
$$

$$
u_{\mathrm{n}}
$$

Then the process of constructing similar dependence graphs continues from the final to the initial time interval.

To find a sequence of optimal controls $\mathrm{u}_{1}{ }^{*}, \mathrm{u}_{2}{ }^{*}, \ldots, \mathrm{u}_{\mathrm{n}}{ }^{*}$ by a known initial state $\mathrm{z}_{0}$, it is necessary to carry out the inverse procedure from the first interval to the last one. For this purpose, using the dependence $u_{1}{ }^{*}=\varphi_{1}\left(z_{0}\right)$ on the first interval, determine the value of $u_{1}{ }^{*}$ and use the transition function to calculate the value $\mathrm{z}_{1}{ }^{*}=\psi\left(\mathrm{z}_{0}, \mathrm{u}_{1}{ }^{*}\right)$, the initial value for the second interval. On the second interval the procedure is repeated, i.e., $\mathrm{u}_{2}{ }^{*}=\varphi_{2}\left(\mathrm{z}_{1}{ }^{*}\right)$ и $\mathrm{z}_{2}{ }^{*}=$ $\psi\left(\mathrm{z}_{1}{ }^{*}, \mathrm{u}_{2}{ }^{*}\right)$, etc. is determined.

To explain such a procedure, here is an example from [5].

The control object takes three states $Z=\left\{\sigma_{1}, \sigma_{2}, \sigma_{3}\right\}$, and the control actions on it include two possible values $U=\left\{\alpha_{1}, \alpha_{2}\right\}$. The transient function of the control object $z_{i}=\psi\left(z_{i-1}, u_{i}\right)$ is given in the form of Table 1.

Table 1. Values of the transition function.

\begin{tabular}{|c|c|c|c|}
\hline \multirow{2}{*}{$\mathbf{u}_{\mathbf{i}}$} & \multicolumn{3}{|c|}{$\mathbf{z}_{\mathrm{i}-\mathbf{1}}$} \\
\cline { 2 - 4 } & $\sigma_{1}$ & $\sigma_{2}$ & $\sigma_{3}$ \\
\hline$\alpha_{1}$ & $\mathrm{z}_{\mathrm{i}}=\sigma_{1}$ & $\mathrm{z}_{\mathrm{i}}=\sigma_{3}$ & $\mathrm{z}_{\mathrm{i}}=\sigma_{1}$ \\
\hline$\alpha_{2}$ & $\mathrm{z}_{\mathrm{i}}=\sigma_{2}$ & $\mathrm{z}_{\mathrm{i}}=\sigma_{1}$ & $\mathrm{z}_{\mathrm{i}}=\sigma_{3}$ \\
\hline
\end{tabular}

Number of time intervals $n=2$, the fuzzy goal at time $\mathrm{t}==2$ is defined by the membership function $\mu_{\mathrm{A}}\left(\mathrm{z}_{\mathrm{n}}\right)$ taking values: : $\mu_{\mathrm{A}}\left(\sigma_{1}\right)=0.3 ; \mu_{\mathrm{A}}\left(\sigma_{2}\right)=1 ; \mu_{\mathrm{A}}\left(\sigma_{3}\right)=0.8$. The fuzzy constraints on the control actions are given by membership functions $\square 1(u 1)$ for the interval $i=1$ and $\mu_{2}\left(u_{2}\right)$ for the interval $i=2$, taking the following values for the corresponding controls $u_{1} \in$ $\left\{\alpha_{1}, \alpha_{2}\right\}$ and $u_{2} \in\left\{\alpha_{1}, \alpha_{2}\right\}$ and $\mu_{1}\left(\alpha_{1}\right)=0,7 ; \mu_{1}\left(\alpha_{2}\right)=1 ; \mu_{2}\left(\alpha_{1}\right)=1 ; \mu_{2}\left(\alpha_{2}\right)=0.6$. The initial state of the object at time $\mathrm{t}=0$ is $\mathrm{z}_{0}=\sigma_{1}$.

It is required to determine the sequence of control actions $\mathrm{u}_{1}{ }^{*}, \mathrm{u}_{2}{ }^{*}$, providing the maximum degree of achievement of a given fuzzy goal for given fuzzy constraints [6].

To solve the problem using (6), we define the membership function of the fuzzy solution

$$
\mu_{\mathrm{R}}\left(z_{2}\right)=\min \left\{\mu_{\mathrm{A}}\left(z_{2}\right), \mu_{2}\left(u_{2}\right)\right\}
$$

Results and discussion

Based on the method of dynamic programming, we set the values $\mathrm{u}_{1}{ }^{*}, \mathrm{u}_{2}{ }^{*}$, providing max $\mu_{R}\left(z_{2}\right)$. To this end, for interval $i=2$, we define functions $f_{1}\left(z_{1}\right)$ and $u_{2}==\varphi_{2}\left(z_{1}\right)$, and for interval $i=1$ we define functions $f_{2}\left(z_{0}\right)$ and $u_{1}=\varphi_{1}\left(z_{0}\right)$ that provide the maximum degree of goal achievement. Then, using the given initial state $\mathrm{z} 0$ and the obtained functions $\mathrm{u}_{1}$ and $\mathrm{u}_{2}$, we set the required values $\mathrm{u}_{1}{ }^{*}, \mathrm{u}_{2}{ }^{*}$.

Thus, we implement the following sequence of actions. Let's define the function 


$$
\begin{array}{r}
f_{1}\left(z_{1}\right)=\max \mu_{\mathrm{R}}\left(z_{2}\right)=\max \min \left\{\mu_{\mathrm{A}}\left(z_{2}\right), \mu_{2}\left(u_{2}\right)\right\} \\
=\max \min \left\{\mu_{\mathrm{A}}\left[\psi\left(z_{1}, u_{2}\right)\right], \mu_{2}\left(u_{2}\right)\right\} . \\
u_{2} \quad u_{2}
\end{array}
$$

By trying different $\mathrm{z}_{1} \in \mathrm{Z}$ and $\mathrm{u}_{2} \in \mathrm{U}$ we set the table of function values $\psi$, and according to the initial data - the values of $\mu_{\mathrm{A}}, \mu_{2}$ From the obtained $\mu_{\mathrm{A}}$ and $\mu_{2}$ we choose the minimum values defining the function $\mu_{\mathrm{R}}\left(\mathrm{z}_{2}\right)=\mu_{\mathrm{R}}\left[\psi\left(\mathrm{z}_{1}, \mathrm{u}_{2}\right)\right]$, which we then maximize by $u_{2}$. As a result, we obtain the following values of the function $\mathrm{f}_{1}\left(\mathrm{z}_{1}\right)$ for all admissible values of $\mathrm{z}_{1} \in\left\{\sigma_{1}, \sigma_{2}, \sigma_{3}\right\}: \mathrm{f}_{1}\left(\sigma_{1}\right)=0.6 ; \mathrm{f}_{1}\left(\sigma_{2}\right)=0.8 ; \mathrm{f}_{1}\left(\sigma_{3}\right)=0.6$.

Simultaneously, we form a function $\mathrm{u}_{2}=\varphi_{2}\left(\mathrm{z}_{1}\right)$ which establishes the following relations between the admissible values $\mathrm{z}_{1} \in\left\{\sigma_{1}, \sigma_{2}, \sigma_{3}\right\}$ and $\mathrm{u}_{2} \in\left\{\alpha_{1}, \alpha_{2}\right\}: \alpha_{2}=\varphi_{2}\left(\sigma_{1}\right), \alpha_{1}=\varphi_{2}\left(\sigma_{2}\right), \alpha_{2}$ $==\varphi_{2}\left(\sigma_{3}\right)$.

Let's define the function

$$
\begin{array}{cc}
f_{2}\left(z_{0}\right)=\max f_{1}\left(z_{1}\right)=\max & f_{1}\left[\psi\left(z_{0}, u_{1}\right)\right] . \\
u_{1} & u_{1}
\end{array}
$$

For this purpose, by trying different $\mathrm{z}_{0} \in \mathrm{Z}$ and $\mathrm{u}_{1} \in$ from Table 1 , we determine the corresponding values of $\psi$. Using the obtained values $\psi\left(z_{0}, u_{1}\right)=z_{1}$ and $f_{1}\left(z_{1}\right)$ we set $f_{1}\left[\psi\left(z_{0}\right.\right.$, $\mathrm{u}_{1}$ )], which we then maximize over $\mathrm{u}_{1}$. As a result, we obtain the following function values $\mathrm{f}_{2}\left(\mathrm{z}_{0}\right): \mathrm{f}_{2}\left(\sigma_{1}\right)=0.8 ; \mathrm{f}_{2}\left(\sigma_{2}\right)==0.6 ; \mathrm{f}_{2}\left(\sigma_{3}\right)=0.6$.

At the same time, we form the function $u_{1}=\varphi_{1}\left(z_{0}\right): \alpha_{2}=\varphi_{1}\left(\sigma_{1}\right), \alpha_{1}$ or $\alpha_{2}=\varphi_{1}\left(\sigma_{2}\right), \alpha_{1}$ or $\alpha_{2}=\varphi_{1}\left(\sigma_{3}\right)$.

The generated functions $\varphi_{1}$ and $\varphi_{2}$ allow to determine the required values of $u_{1}{ }^{*}, u_{2}{ }^{*}$. To do this, substitute the value of the initial state $z_{0}=\sigma_{1}$ into the function $u_{1}=\varphi_{1}\left(z_{0}\right)$ and obtain $\mathrm{u}_{1}{ }^{*}=$

$=\alpha_{2}=\varphi_{1}\left(\sigma_{1}\right)$.

Then we determine $\mathrm{z}_{1}{ }^{*}=\psi\left(\mathrm{z}_{0}, \mathrm{u}_{1}{ }^{*}\right)=\psi\left(\sigma_{1}, \alpha_{2}\right)=\sigma_{2}$ and consequently $\mathrm{u}_{2}{ }^{*}=\varphi_{2}\left(\mathrm{z}_{1}{ }^{*}\right)=$ $\varphi_{2}\left(\sigma_{2}\right)=\alpha_{1}$

\section{Conclusions}

Thus, we obtain the following optimal control actions: $\mathrm{u} 1 *=\square 2, \mathrm{u} 2 *=\square 1$. For successful problem solving under conditions of fuzzy information, it is necessary to compile a knowledge base for transportation processes, which would allow to take into account and process fuzzy perceptions and judgments of transport company managers about the ongoing processes with the help of more adequate models. Transport processes are tasks with fuzzy conditions to the maximum extent correspond to most real-world problems that may arise in the management process in various situations where it is necessary to make decisions in the shortest possible time with very fuzzy source information, characterized not by specific quantitative data about the control objective, but by fairly general qualitative representations of the given conditions.

\section{References}

1. R. Bellman, Dynamic Programming (1960)

2. R., Bellman, L. Zadeh, Decision-making in vague conditions (1976) 
3. V. A. Boldinov, V. A. Bukhalev, A. A. Skrynnikov, Izvestiya RAS Theory and control systems, 4, 18 (2020)

4. D. A. Pospelov, Situational management: theory and practice (1986)

5. Problems of program-target planning and management (1981)

6. S. G. Pushkov, Izvestiya RAS Theory and control systems, 2, 25 (2021)

7. Reality and forecasts of artificial intelligence (1987)

8. T. Saati, Decision-making. Hierarchy analysis method (1993)

9. A. N. Asaul, The theory and practice of acceptance development and implementation of administrative decisions in business, 304 (2014)

10. V. I. Zhurko, Social technologies of development and acceptance of administrative decisions, 125 (2015)

11. M. G. Kovtunovich, The Bulletin of Academy of the Right and Management, 44, 159 (2016)

12. A. S. Kozlov, Decision-making technologies, 147 (2015) 